\title{
Research on Secure Storage of Internet of Things Information Records Based on Block Chain Technology
}

\author{
Yang Ying \\ Chongqing Vocational Institute of Engineering, Chongqing, 400000, China
}

Keywords: Block chain technology, Information records of the internet of things, Secure storage

\begin{abstract}
With the increase of Iot equipment, different problems such as coordination and cooperation between equipment and equipment still need to be solved. In order to better put forward the corresponding scheme, in-depth analysis and research on this technology are also needed. With the rapid development of the big data era, the Internet of Things has become an important content that cannot be ignored in people's life. How to ensure the security and stability of all information transmission and information recording in the Internet of Things system, so that people can not only ensure the information to be stored for a long time, but also prevent the information in the Internet of Things from being stored for a long time because hackers or other organizations steal the information, and even bring negative effects to the active competition in the market. Combining blockchain and container technology, this paper proposes a secure storage scheme for Internet node access information, which aims to ensure the information storage efficiency and security of the Internet of things. So that the real data of information interaction between nodes of the Internet of things can be saved in the storage structure under the chain. It effectively realizes the security of IOT nodes, and reduces the difficulty of information acquisition and traceability after malicious attacks on IOT information.
\end{abstract}

\section{Introduction}

In recent years, with the rapid development of the Internet, more and more people use the Internet and the Internet of Things. The Internet of Things has already entered people's life. In the near future, the arrival of $5 \mathrm{~g}$ era will make people use the Internet of Things, and its actual use effect will gradually increase. However, how to speed up the use of IoT and increase Internet vendors' awareness of IoT requires linking block chain technology with Internet of Things information recording and safe storage to realize cloud platform's supervision and control of IoT equipment. Working together in various devices can provide more convenience for users in their daily life and work, and can also gradually reveal a series of problems existing in IoT itself. This includes a series of problems such as the safety of equipment, personal privacy and the rigidity of traditional IoT equipment. Among them, the security of devices and personal security are particularly important. The main source of these problems is that traditional IOT devices have certain vulnerabilities. When users use it, their own security awareness is low. Hackers can easily crack IOT devices through relevant software tools. In terms of personal privacy, the disclosure is very serious. However, the centralized management architecture contained in traditional IOT devices is difficult to obtain evidence and trace the stolen or attacked information. The integration of blockchain technology and container technology can improve the use effect of IOT devices and improve the security of information management.

\section{Iot Information Record Security Storage Scheme}

With the rapid development of science and technology, the development speed of block chain technology has become faster and faster in recent years. As a storage structure with the characteristics of anonymity, decentralization, and untrusted third parties, block chain has been used in the information security of Internet of Things as the basis for building a feasible computing platform. In the traditional application of block chain, it mainly uses bitcoin and Ethernet to 
calculate the consensus layer contained in itself, which requires a lot of calculation force in the calculation process. Compared with the traditional IoT equipment, the brand-new block chain technology gradually reduces the equipment consumption. Traditional IOT devices in blockchain nodes need to prove mechanism, which makes the workload gradually increase. The consumption of resources is also gradually increasing, which can not be directly deployed in traditional IOT nodes with relatively low computing power, making IOT in information security storage, its quality is poor.

However, with the new blockchain technology, IOT device node information can be written and stored, which can be applied in blockchain network script, and then a new transaction processing method can be generated. The algorithm has basically completed the actual requirement of deblocking chain function. Whether using Python Flask framework or Python Flask framework, the Rest API function contained in the framework is exposed in this form. Its purpose is to gradually improve the quality of information security storage by block chain technology, so that device nodes can use common HTTP request methods. For example, through get and post, data submission, transaction data and inquiry of data, transaction information and node information contained in the block chain network are initiated into the upper block chain network. As users, they can also control network nodes through API. There are a large number of calculation processes in the block chain. The calculation process itself is deployed at the server. Its purpose is to reduce the pressure of Iot equipment currently used and ensure the robustness of the overall network. In the brand-new block chain technology Internet of Things information technology security, because information storage transactions only need to briefly describe the information and save the relevant hash value, the information can be traced back, while the most important information is saved in the equipment under the chain, further containerizing the block chain nodes. Its purpose is to facilitate the overall data migration, node deployment and trial when the system is replaced. Use port mapping technology to map apl contained in nodes.

The data records between IoT equipment and nodes and container nodes are transmitted nearby in a data exchange format of lighter magnitude. Through a large number of experiments, it is proved that the data information and information development have good readability and are convenient for data exchange and data analysis on different platforms. In the common block chain technology, when attacking Iot equipment, the main attack method is that hackers use scanning software to discover the ip of the equipment, and to steal and tamper with the equipment information according to the loopholes existing in the equipment. In the new scheme, Internet of things security protocol is directly connected to the network, which ensures that IOT devices in the access to the blockchain network security network, its security system is high, and can be assigned to a new container node.

The same LAN can be obtained by mapping the ports contained in this container node to brandnew IoT equipment. This not only facilitates the connection between the Iot equipment and the container, facilitates the information transmission, but also enables the Iot equipment to have corresponding scripts in the process of writing in advance. After the container is connected, its information status can meet people's needs for IoT equipment. When sending the device, it also contains the basic state information, which makes the container node register after completing the blockchain transaction node registration. The container node and the IOT node can complete the data interaction device, and send the relevant data through HTTP request. The container node in the blockchain will make a new judgment and record of the IOT device status in the corresponding time period. Once the IOT node in the information recording security system of the blockchain Internet of things is attacked, the corresponding transaction node writes IOT information interaction records in the chain storage structure of the blockchain, and spreads to the whole blockchain network node, and the container node will automatically shut down under the corresponding circumstances. There is a monitoring system in the whole security system and monitoring the status of containers and container hosts, which can discover possible or existing security problems in the network in time and help users to maintain and manage the network in time. 


\section{Security Analysis}

(1) Tampering prevention and identity authentication

In the Internet of Things information recording security of block chain technology, it has tamperproof function. All information stored in the security system chain can be written into the block only after being confirmed by nodes in the network, and all records, whether funds or transactions, contain time stamps. In other words, each transaction can be rearranged according to the time stamp sequence in the block, and trust can be established in the block chain system according to the distributed consensus mechanism contained in the security system. It is based on a reliable cryptographic algorithm and does not need to rely on a trusted third party. Once the data is written into the block chain, it cannot be tampered with and its preservation effect is relatively safe. However, each block in the chain structure of the blockchain will be recorded on the previous block, and promote the hash value of the block data quality. If you want to modify the data that has been stored in the blockchain, you need to ensure $51 \%$ of the computing power in the whole network, which is almost impossible for the relatively large IOT to illegally change the data information contained in the blockchain. If the relevant information is obtained through illegal attack, it will not only cause the blockchain structure in the security system to record the content of the attack, but also ensure the security of the information. Because the original IOT node is stored in the blockchain structure on the security system, the hash value of information record directly modifies the original information, which will also cause the hash value in the block to change. For this reason, it also ensures that the information of IOT node itself can not be tampered. In the information recording of the Internet of things based on blockchain technology, IOT devices of security system and transaction nodes of blockchain are connected by IOT protocol. Whether using Bluetooth or Zig Bee, the upper layer can record the information when using the network. Each device corresponds to a node. Using this transaction node can improve the overall effect of verification, using the verification and consensus mechanism of blockchain, can effectively avoid illegal intrusion, and also can avoid malicious contacts in blockchain accessing IOT by borrowing.

Compared with the traditional IoT, its security is higher and safer. As the brand-new block chain technology combines the traditional IoT technology, it has a qualitative improvement on the overall security of the network. The specific security improvement includes the following points: in the traditional IoT, the incomplete information record can protect the information and ensure the storage security of the information, but it needs to rely on a third party and has low reliability. However, the use of a new block chain security system can not only ensure the integrity of information records, but also meet the current actual needs for information in privacy protection, secure storage and trusted third parties. By virtue of the characteristics of the block chain itself, such as de-centralization, information disclosure, transparency, security and transparency, and difficulty in tampering after writing blocks, it can not only ensure the security of equipment information storage, but also check all information in the security system. The modification of information records needs to go through more than half of the nodes, in order to confirm or increase the difficulty of attackers tampering with the information of the Internet of things in block technology and ensure the integrity of information records. The authority management of identity and consensus algorithm can help the system effectively identify the involvement of illegal contacts. For illegal nodes, their chain structure based on blockchain can more easily obtain relevant electronic certificates. Different from other technologies, in the process of information security storage of the Internet of things using blockchain technology, compared with the traditional information, it will be better than the traditional security system, and has a very obvious breakthrough and innovation.

(2) Compared with the traditional IoT equipment, the new equipment has the advantages

Compared with the original IoT information record security storage system built by container technology, its main innovations are as follows: First, using the characteristics of block chain technology itself, such as de-centralization, multi-point confirmation, difficult tampering and multiparty consensus, it can solve the different contents and problems of centralized architecture, 
equipment security and privacy protection in IoT. Using the block chain can be simply understood as a distributed database, that is to say, each data is stored in a separate database to consider, which is called transaction. According to a certain order, different data can be placed in a fixed time stamp each time, and each piece of data contains this piece of data and the hash value generated after the previous piece of data is recorded in the block chain, which requires multiple data blocks to form a complete chain of data blocks. The existence of its data block chain can not only improve the storage effect, but also ensure the integrity and traceability of information. Therefore, the block chain nodes can be stored in the server in the form of containers, so that the overall system platform of the deblocking chain can be continuously deployed across platforms, and the overall characteristics of the test are improved. Giving the consensus mechanism in the block chain to the server can not only ensure the robustness of the overall server, but also ensure the overall effect of the block chain technology in application. Not only that, in the Internet of Things information record using the block chain technology, a visual web platform is also realized, and then the IoT node and the block chain node exchange information, which not only facilitates the information collection of the IoT equipment by the Web, but also can be managed and monitored by using corresponding container management software in the containerized block chain node. As users, they can directly observe the overall state of the network on the platform, and quickly counterattack those who illegally invade the network to ensure that all information storage effects in the network system can meet people's actual needs for information security.

\section{Conclusion}

To sum up, the Internet of Things information recording security storage system based on block chain technology uses brand-new IoT information recording, which provides a secure distributed storage mechanism for information and enables the information recording function of Iot equipment to be fully and safely used. In the chain structure, the use of container technology not only facilitates system deployment and information migration, but also realizes molecules of consensus layer and IoT layer in the block chain. The relatively difficult calculation is directly left to the server to ensure the overall security and robustness of the network when using the blockchain technology in the Internet of things information storage process. At the same time, it can also ensure the overall effect of information in sharing, use and analysis. In the security system, there are still many problems to be solved. The purpose is to gradually improve the effect of blockchain technology in the secure storage of Internet of things information records.

\section{References}

[1] Feng Wei, Hu bin, Li Qisheng, Chen Junrong, Chen Yahui. Research on the supervision of IOT financial movable property financing based on block IOT technology [J]. Guangxi quality supervision guide, 2019 (11): 185-187

[2] Fang Shihong, Peng Xiyu. Secure storage of Internet of things information records based on blockchain technology [J]. Journal Of Southwest University For Nationalities (Natural Science Edition), 2019,45 (03): 284-289 